OCHA-PP-248

NIIG-DP-05-1

July 2005

\title{
Hybrid Textures of Neutrinos
}

\author{
Satoru Kaneko ${ }^{1}$, Hideyuki Sawanaka ${ }^{2}$ and Morimitsu Tanimoto ${ }^{3}$ \\ ${ }^{1}$ Physics Department, Ochanomizu University, Tokyo 112-8610, Japan \\ ${ }^{2}$ Graduate School of Science and Technology, Niigata University, Niigata 950-2181, Japan \\ ${ }^{3}$ Physics Department, Niigata University, Niigata 950-2181, Japan
}

\begin{abstract}
We present numerical and comprehensive analyses of the sixty hybrid textures of neutrinos, which have an equality of matrix elements and one zero. These textures are possibly derived from the discrete symmetry. Only six textures among sixty ones are excluded by the present experimental data. Since there are many textures which give similar predictions, the textures are classified based on the numerical results. The neutrinoless double beta decay is also examined in these textures. Our results suggest that there remain still rich structures of the neutrino mass matrix in the phenomenological point of view.
\end{abstract}




\section{Introduction}

The neutrino mass-squared differences and the mixing angles are now quite well determined by the neutrino oscillation experiments $[1,2,3]$. The third mixing angle, represented by the matrix element $U_{e 3}$ of the lepton mixing matrix $U$ (MNS matrix [4]), is constrained to be small by the non-observation of neutrino oscillations at the CHOOZ experiment [5].

These results indicate the bi-large flavor mixing in the lepton sector. It is therefore important to investigate how the textures of lepton mass matrices can link up with the observables of the flavor mixings. Many authors studied the texture zeros $[6,7,8,9]$, which may follow from the flavor symmetry. On the other hand, one finds some relations among the non-zero mass matrix elements in the discrete symmetry of the flavor. This fact suggests that the texture zero analyses is not enough to reveal some underlying flavor symmetry.

For example, in the $e, \mu, \tau$ basis, one finds the following symmetric mass matrices of neutrinos, where there are two entries with same values and one zero,

$$
\left(\begin{array}{lll}
a & c & d \\
& b & 0 \\
& & b
\end{array}\right), \quad\left(\begin{array}{lll}
0 & c & d \\
& a & b \\
& & a
\end{array}\right),
$$

both are presented in the quaternion family symmetry, $Q_{8}[10]$ and the latter is given in the $S_{3}$ symmetry [11]. The variant of these textures is also discussed in ref. [12]. We call this type texture as the "Hybrid" texture, which has an equality of matrix elements and one zero.

The analytical study of various structures of the neutrino mass matrix was presented systematically by Frigerio and Smirnov [13], who also discussed the case of equalities of matrix elements. The textures for the Dirac neutrinos also discussed in ref. [14]. However, numerical and comprehensive analyses have not been given.

In this paper, we present numerical analyses of the sixty hybrid textures, which have two equal mass matrix elements with one zero. Our analyses include textures in the previous studies in refs. $[10,11]$. Our results are consistent with their results.

The hybrid textures are discussed in section 2, and numerical results are presented in section 3. Section 4 is devoted to the summary. 


\section{Hybrid Textures}

Let us construct the neutrino mass matrix in terms of neutrino mass eigenvalues $m_{1}, m_{2}, m_{3}$, mixing angles and $\mathrm{CP}$ violating phases. Neutrino mass matrix $M_{\nu}$ in the basis where the charged lepton mass matrix is diagonal (flavor basis) is given as follows:

$$
M_{\nu}=P U^{*} M_{\text {diagonal }} U^{\dagger} P
$$

where $M_{\text {diagonal }}$ and $P$ are the diagonal mass matrix and the diagonal phase matrix, respectively:

$$
M_{\text {diagonal }}=\left(\begin{array}{ccc}
\lambda_{1} & 0 & 0 \\
0 & \lambda_{2} & 0 \\
0 & 0 & \lambda_{3}
\end{array}\right), \quad P=\left(\begin{array}{ccc}
e^{i \phi_{e}} & 0 & 0 \\
0 & e^{i \phi_{\mu}} & 0 \\
0 & 0 & e^{i \phi_{\tau}}
\end{array}\right)
$$

where $\lambda_{i}(i=1,2,3)$ are the complex mass eigenvalues including Majorana phases, so three neutrino masses $m_{i}$ are given as the absolute values of $\lambda_{i}$. On the other hand, $\phi_{i}(i=e, \mu, \tau)$ are unphysical phases depending on the phase convention. The MNS mixing matrix $U$ is parametrized as

$$
U=\left(\begin{array}{ccc}
c_{13} c_{12} & c_{13} s_{12} & s_{13} e^{-i \delta} \\
-c_{23} s_{12}-s_{23} s_{13} c_{12} e^{i \delta} & c_{23} c_{12}-s_{23} s_{13} s_{12} e^{i \delta} & s_{23} c_{13} \\
s_{23} s_{12}-c_{23} s_{13} c_{12} e^{i \delta} & -s_{23} c_{12}-c_{23} s_{13} s_{12} e^{i \delta} & c_{23} c_{13}
\end{array}\right)
$$

where $c_{i j}$ and $s_{i j}$ denote $\cos \theta_{i j}$ and $\sin \theta_{i j}$, respectively. Then, the neutrino mass matrix elements $\left(M_{\nu}\right)_{\alpha \beta}$ are given in the flavor basis as

$$
\left(M_{\nu}\right)_{\alpha \beta}=e^{i\left(\phi_{\alpha}+\phi_{\beta}\right)} \sum_{f}^{3} U_{\alpha f}^{*} U_{\beta f}^{*} \lambda_{f}=e^{i\left(\phi_{\alpha}+\phi_{\beta}\right)}\left(U_{\alpha 1}^{*} U_{\beta 1}^{*} \lambda_{1}+U_{\alpha 2}^{*} U_{\beta 2}^{*} \lambda_{2}+U_{\alpha 3}^{*} U_{\beta 3}^{*} \lambda_{3}\right) .
$$

By use of these mass matrix elements, we can analyze the sixty cases in terms of mass eigenvalues and mixings. These cases are combinations of the one zero textures and the equal elements: (i) fifteen cases of the equal elements (Type $\mathrm{A} \sim \mathrm{O})\left(M_{\nu}\right)_{\alpha \beta}=\left(M_{\nu}\right)_{\gamma \delta}$, (ii) six cases of one zero (Type I $\sim \mathrm{VI})\left(M_{\nu}\right)_{\alpha \beta}=0$. These combinations give ninety textures, however, among them, thirty textures have two zeros, which have been studied in details $[6,7,8,9]$. Therefore, we study numerically in the sixty textures, which are summarized in Table 1. 


\section{Numerical Results}

Since the textures have the conditions $\left(M_{\nu}\right)_{\alpha \beta}=0$ and $\left(M_{\nu}\right)_{i j}=\left(M_{\nu}\right)_{k \ell}$, we can get the ratios of mass eigenvalues by solving two equations as follows [8]:

$$
\begin{aligned}
& \frac{\lambda_{1}}{\lambda_{2}}=\frac{\left(U_{i 3}^{*} U_{j 3}^{*}-Q U_{k 3}^{*} U_{\ell 3}^{*}\right) U_{\alpha 2}^{*} U_{\beta 2}^{*}-\left(U_{i 2}^{*} U_{j 2}^{*}-Q U_{k 2}^{*} U_{\ell 2}^{*}\right) U_{\alpha 3}^{*} U_{\beta 3}^{*}}{\left(U_{i 1}^{*} U_{j 1}^{*}-Q U_{k 1}^{*} U_{\ell 1}^{*}\right) U_{\alpha 3}^{*} U_{\beta 3}^{*}-\left(U_{i 3}^{*} U_{j 3}^{*}-Q U_{k 3}^{*} U_{\ell 3}^{*}\right) U_{\alpha 1}^{*} U_{\beta 1}^{*}}, \\
& \frac{\lambda_{3}}{\lambda_{2}}=\frac{\left(U_{i 1}^{*} U_{j 1}^{*}-Q U_{k 1}^{*} U_{\ell 1}^{*}\right) U_{\alpha 2}^{*} U_{\beta 2}^{*}-\left(U_{i 2}^{*} U_{j 2}^{*}-Q U_{k 2}^{*} U_{\ell 2}^{*}\right) U_{\alpha 1}^{*} U_{\beta 1}^{*}}{\left(U_{i 1}^{*} U_{j 1}^{*}-Q U_{k 1}^{*} U_{\ell 1}^{*}\right) U_{\alpha 3}^{*} U_{\beta 3}^{*}-\left(U_{i 3}^{*} U_{j 3}^{*}-Q U_{k 3}^{*} U_{\ell 3}^{*}\right) U_{\alpha 1}^{*} U_{\beta 1}^{*}},
\end{aligned}
$$

where $Q \equiv e^{i \varphi}=e^{i\left(\phi_{k}+\phi_{\ell}-\phi_{i}-\phi_{j}\right)}$. Taking absolute values of these ratios, we get the neutrino mass ratios, $m_{1} / m_{2}$ and $m_{3} / m_{2}$. Therefore mass ratios are given in terms of $\theta_{12}, \theta_{23}, \theta_{13}, \delta$ and the unknown phase $\varphi$. Absolute values of neutrino masses are fixed by putting the experimental data $\Delta m_{\text {atm }}^{2}$ and $\Delta m_{\text {sun }}^{2}$.

We present the scattered plots of the allowed region in the $\sin ^{2} 2 \theta_{23}-\left|U_{e 3}\right|$ plane and the $m_{2}-m_{3}$ plane, in which the experimental data are taken in the 90\% CL limit $[2,15]$ :

$$
\begin{array}{r}
0.33 \leq \tan ^{2} \theta_{\text {sun }} \leq 0.49, \quad 7.7 \times 10^{-5} \leq \Delta m_{\text {sun }}^{2} \leq 8.8 \times 10^{-5} \mathrm{eV}^{2} \\
0.92 \leq \sin ^{2} 2 \theta_{\mathrm{atm}}, \quad 1.5 \times 10^{-3} \leq \Delta m_{\mathrm{atm}}^{2} \leq 3.4 \times 10^{-3} \mathrm{eV}^{2}
\end{array}
$$

The bound obtained by the reactor neutrinos [5] $\theta_{\text {reactor }} \leq 12^{\circ}$ is also taken in our study.

We classify the textures based on the predicted mixings $\theta_{23}$ and $\left|U_{e 3}\right|$. We cannot distinguish the textures by the mixing $\theta_{12}$ at the present stage of the experimental data:

- The predicted mixings of the eighteen textures cover whole experimental allowed region: A-I, A-II, A-III, B-I, B-II, B-III, D-V, D-VI, E-II, F-I, I-I, I-II, L-I, L-II, L-IV, O-I, O-II, O-IV

- The allowed points of the twenty-two textures are very few:

C-I, C-II, C-III, D-III, E-V, E-VI, F-V, F-VI, G-III, G-V, G-VI, H-III, H-V, H-VI, J-III, J-VI, K-III, K-VI, M-III, M-V, N-III, N-V

- The $\sin ^{2} 2 \theta_{23}$ has the lower bound 0.99 : 
C-IV

- The $\left|U_{e 3}\right|$ of the six textures has the lower bound, which increases $\operatorname{as~}^{2} \sin ^{2} 2 \theta_{23}$ increases: A-VI, B-V, G-II, H-I, L-VI, O-V

The lower bound $\left|U_{e 3}\right| \geq 0.03$ is obtained in A-VI and B-V, and $\left|U_{e 3}\right| \geq 0.04$ is clearly predicted in G-II, H-I. The bound $\left|U_{e 3}\right| \geq 0.05$ is roughly obtained although the allowed points are few in L-VI and $\mathrm{O}-\mathrm{V}$.

- The lower bound of $\left|U_{e 3}\right|$ decreases as $\sin ^{2} 2 \theta_{23}$ increases in the seven textures:

D-IV, I-V, I-VI, J-II, K-I, M-II, N-I,

in which $\left|U_{e 3}\right|=0$ is allowed at $\sin ^{2} 2 \theta_{23}=1$ except D-IV. The texture D-IV has the lower bound $\left|U_{e 3}\right| \geq 0.001$.

- The six textures are excluded by the experimental data:

E-IV, F-IV, J-IV, K-IV, M-IV, N-IV

The mass patterns are summarized in Table 2, in which NH, IH and DL denote the normal mass hierarchy, inverted one and quasi-degenerate masses, respectively, and ALL means including $\mathrm{NH}, \mathrm{IH}$ and DL.

For the typical textures in each group, the allowed regions are shown by scattered plots in both $\sin ^{2} 2 \theta_{23}-\left|U_{e 3}\right|$ plane and the $m_{2}-m_{3}$ plane in fig. $1 \sim$ fig. 5 , where 200,000 $(1,000,000)$ parameter sets are produced randomly for A-I, G-II, I-V (C-III, C-IV) and only experimental allowed sets are plotted.

The texture A-I is a typical one, which leads to the normal hierarchy of the neutrino masses mainly, but the quasi-degenerate spectrum is also allowed, as seen in fig. 1. The predicted mixings cover all experimental allowed region on the $\sin ^{2} 2 \theta_{12}-\left|U_{e 3}\right|$ plane as well as on the $\sin ^{2} 2 \theta_{23}-\left|U_{e 3}\right|$ plane. The texture C-III is a typical one, which leads to the inverted hierarchy of the neutrino masses mainly as seen in fig. 2, in which 1,000,000 parameter sets are put randomly since the allowed points are very few. The texture C-IV gives a specific mass hierarchy and 
mixing angle $\theta_{23}$ as seen in fig. 3 , on the other hand, the predicted $\theta_{12}$ covers whole experimental allowed region. The texture G-II is a typical one, which gives also a specific mass hierarchy and the clear lower bound of $\left|U_{e 3}\right|$ as seen in fig. 4. The texture I-V is a typical one, which leads to the inverted mass hierarchy of neutrino masses. The prediction excludes the specific region on the $\sin ^{2} 2 \theta_{23}-\left|U_{e 3}\right|$ plane as seen in fig. 5. We also show the relative magnitudes of the matrix elements in the unit of $\lambda \simeq 0.2$ for these typical textures in Table 3 .

It may be helpful to see which future data might rule out these textures. If the inverted mass hierarchy is shown to be realized by Nature, the textures of C-IV and G-II are ruled out. On the contrary, if mass spectrum is the normal hierarchy, the textures of C-III and I-V are ruled out. Finding $\sin ^{2} 2 \theta_{23}<0.98$ and $\left|U_{e 3}\right|<0.04$ rule out the texture C-IV and G-II, respectively.

Our numerical results can be understandable in the following analytical studies by using eq.(6). For the texture C-IV, the mass ratios $m_{1} / m_{2}$ and $m_{3} / m_{2}$ are given as

$$
\begin{aligned}
& \frac{m_{1}}{m_{2}}=\left|\frac{\lambda_{1}}{\lambda_{2}}\right|=\frac{s_{12}^{2}}{c_{12}^{2}} \\
& \frac{m_{3}}{m_{2}}=\left|\frac{\lambda_{3}}{\lambda_{2}}\right|=\frac{\cos 2 \theta_{12}}{c_{12}^{2}}\left|1-\frac{1}{2} s_{13} \tan 2 \theta_{12} \sin 2 \theta_{23} \frac{(1+Q) e^{-i \delta}}{c_{23}^{2} Q-s_{23}^{2}}\right|
\end{aligned}
$$

where the first order of $s_{13}$ is remained and the higher order $s_{13}^{2}$ are neglected. It is found that one obtains $m_{1} / m_{2} \simeq 2 / 3$ and $m_{3} / m_{2} \simeq 1 / 3$ in the limit of $s_{13}=0$ with putting $\theta_{12}=\pi / 6$ and $\theta_{23}=\pi / 4$. The value of $m_{1} / m_{2} \simeq 2 / 3$ indicates the normal mass hierarchy. However, $m_{3} / m_{2} \simeq 1 / 3$ contradicts with the experimental mass ratios, and so it is concluded that $s_{13}=0$ is forbidden. In order to see the $s_{13}$ dependence of the lower bound of $\sin ^{2} 2 \theta_{23}$, we take a parametrization of $\theta_{23}=\pi / 4-\epsilon$. Putting it on eq.(8), we get

$$
\frac{m_{3}}{m_{2}}=\frac{\cos 2 \theta_{12}}{c_{12}^{2}}\left|1-\frac{1}{2} s_{13} \tan 2 \theta_{12} \frac{(1+Q) e^{-i \delta}}{\epsilon(1+Q)+\frac{1}{2}(Q-1)}\right|,
$$

in the first order of $\epsilon$. The largest $m_{3} / m_{2}$ is obtained in the case of $Q=1$ and $e^{-i \delta}=-1$. With these phases, $\epsilon$ is given in terms of $s_{13}$ and $m_{3} / m_{2}$ as

$$
\epsilon=\frac{\tan 2 \theta_{12}}{2} s_{13}\left(\frac{m_{3}}{m_{2}} \frac{c_{12}^{2}}{\cos 2 \theta_{12}}-1\right)^{-1}
$$


Taking the boundary of data in eq.(7), one can get the upper bound of $\epsilon$ for the fixed $s_{13}$. In the case of $s_{13}=0.2$, we obtain $\epsilon=0.04$, which leads to the lower bound $\sin ^{2} 2 \theta_{23}=0.99$. This result is good agreement with the numerical one in fig. 3.

It is more easy to understand numerical results for the texture G-II. The mass ratio $m_{3} / m_{2}$ is given as

$$
\frac{m_{3}}{m_{2}}=\left|\frac{\lambda_{3}}{\lambda_{2}}\right|=\left|\frac{s_{12} s_{23}}{s_{13}\left(c_{12} c_{23}+Q s_{12}\right)}\right|,
$$

which is larger than 1 due to $s_{13} \leq 0.2$. Therefore, this texture corresponds to the normal hierarchy of the mass. The lower bound of $s_{13}$ is given with $Q=1$ as

$$
s_{13} \geq \frac{m_{2}}{m_{3}} \frac{s_{12} s_{23}}{s_{13}\left(c_{12} c_{23}+s_{12}\right)} .
$$

Taking the boundary of data in eq.(7), we obtain the lower bound $s_{13}=0.04$, which is also good agreement with the numerical one in fig. 4.

For the texture I-V, the normal mass hierarchy is not allowed as seen in fig. 5. The mass ratio $m_{3} / m_{2}$ is given as

$$
\frac{m_{3}}{m_{2}}=\left|\frac{c_{23}^{2}\left[\cos 2 \theta_{12} e^{i \delta}-c_{12} s_{12} s_{13}\left(Q+2 \tan \theta_{23}\right)\right]}{s_{23} c_{23} s_{12}^{2} Q e^{i \delta}+c_{12}^{2} s_{23}^{2} e^{i \delta}+s_{12} c_{12} s_{23}^{2} s_{13} Q}\right| .
$$

In the limit of $s_{13}=0$, we have

$$
\frac{m_{3}}{m_{2}}=\left|\frac{c_{23} \cos 2 \theta_{12}}{s_{23}\left(s_{12}^{2} Q+c_{12}^{2} \tan 2 \theta_{23}\right)}\right|
$$

while

$$
\frac{m_{1}}{m_{2}}=\left|\frac{c_{12}^{2} Q+s_{12}^{2} \tan \theta_{23}}{s_{12}^{2} Q+c_{12}^{2} \tan \theta_{23}}\right|
$$

at $s_{13}=0$. It is noticed that the normal hierarchy mass is not allowed for an arbitrary phase $Q$, because $m_{3} / m_{2}$ is not larger than 1 . Actually, in the case of $Q=-1$ and $\theta_{23} \simeq \pi / 4$, neutrino masses are degenerate, on the other hand, taking $Q=-1$ we have $m_{3} / m_{2}<1$, therefore, the inverted mass hierarchy is realized. The situation is not changed even if we include the small $s_{13}$ effect. 

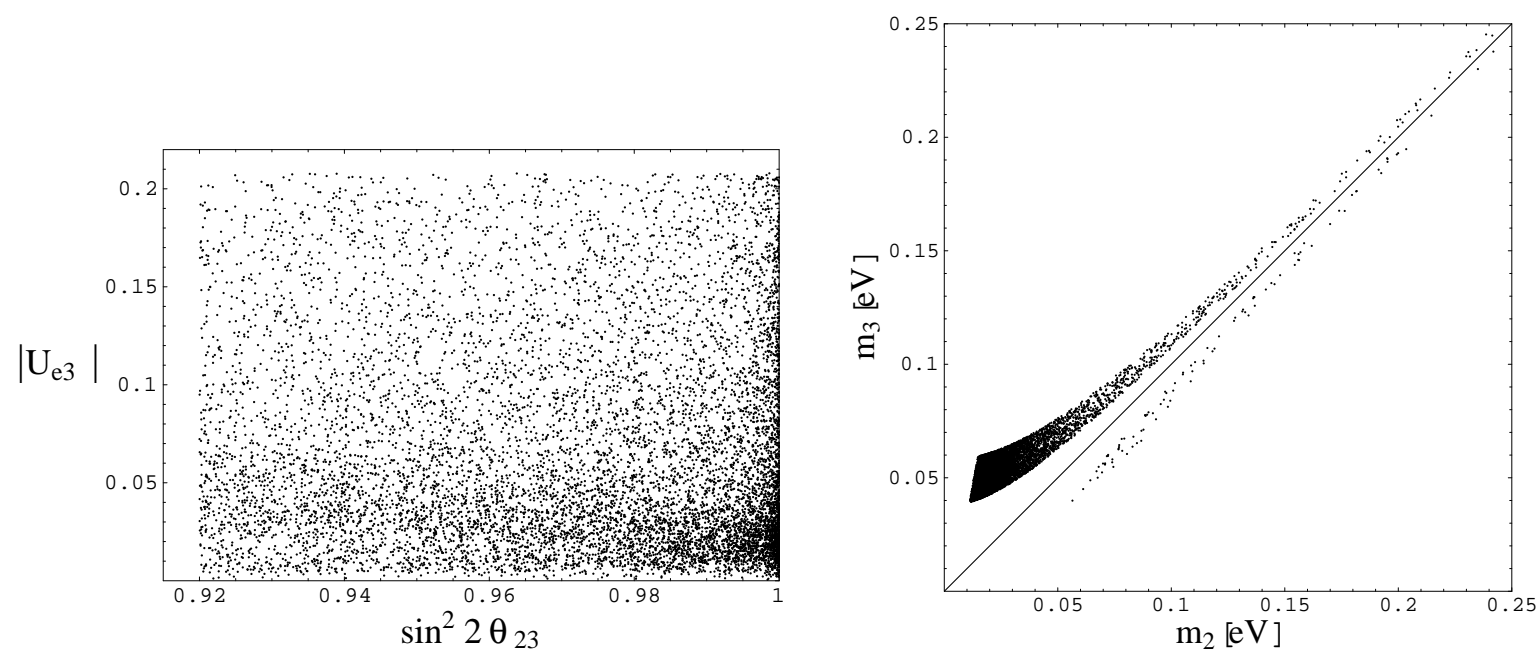

Figure 1: Scattered plot of the allowed region in the texture A-I.
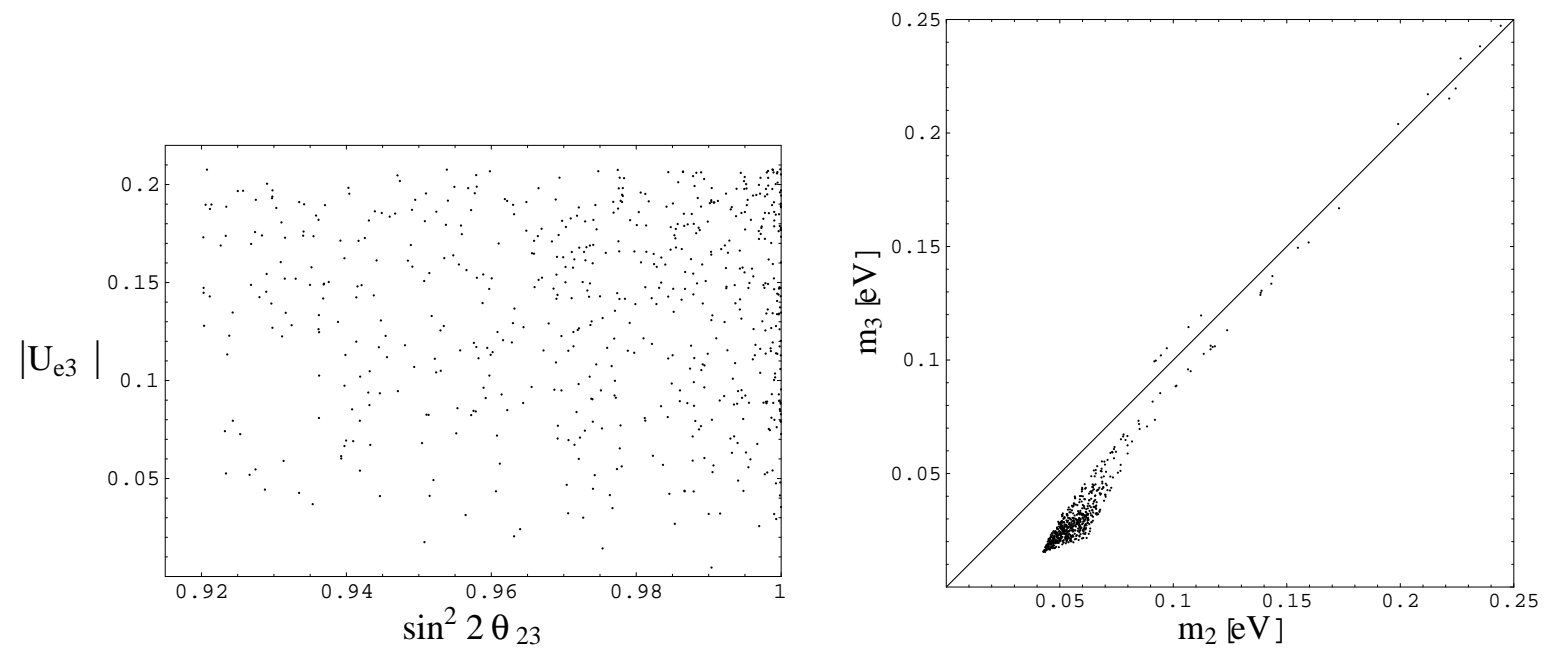

Figure 2: Scattered plot of the allowed region in the texture C-III. 

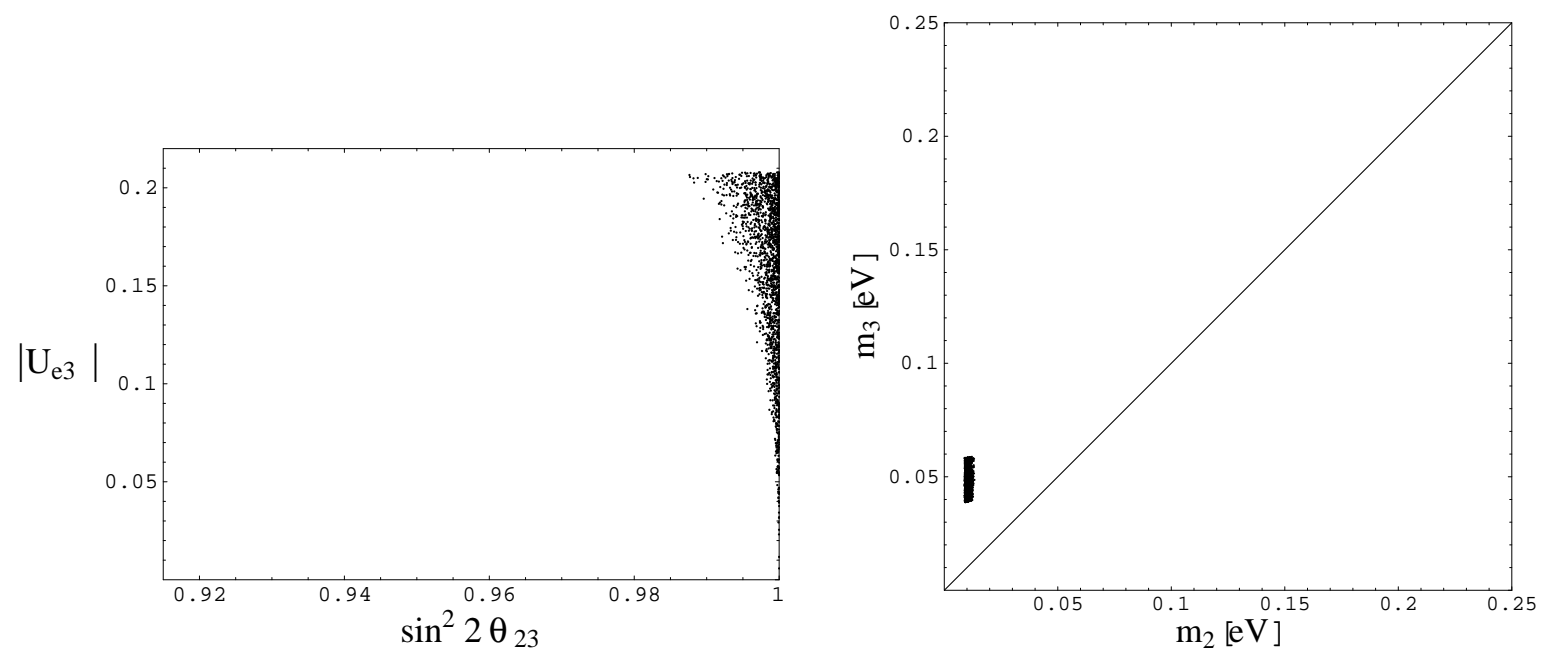

Figure 3: Scattered plot of the allowed region in the texture C-IV.
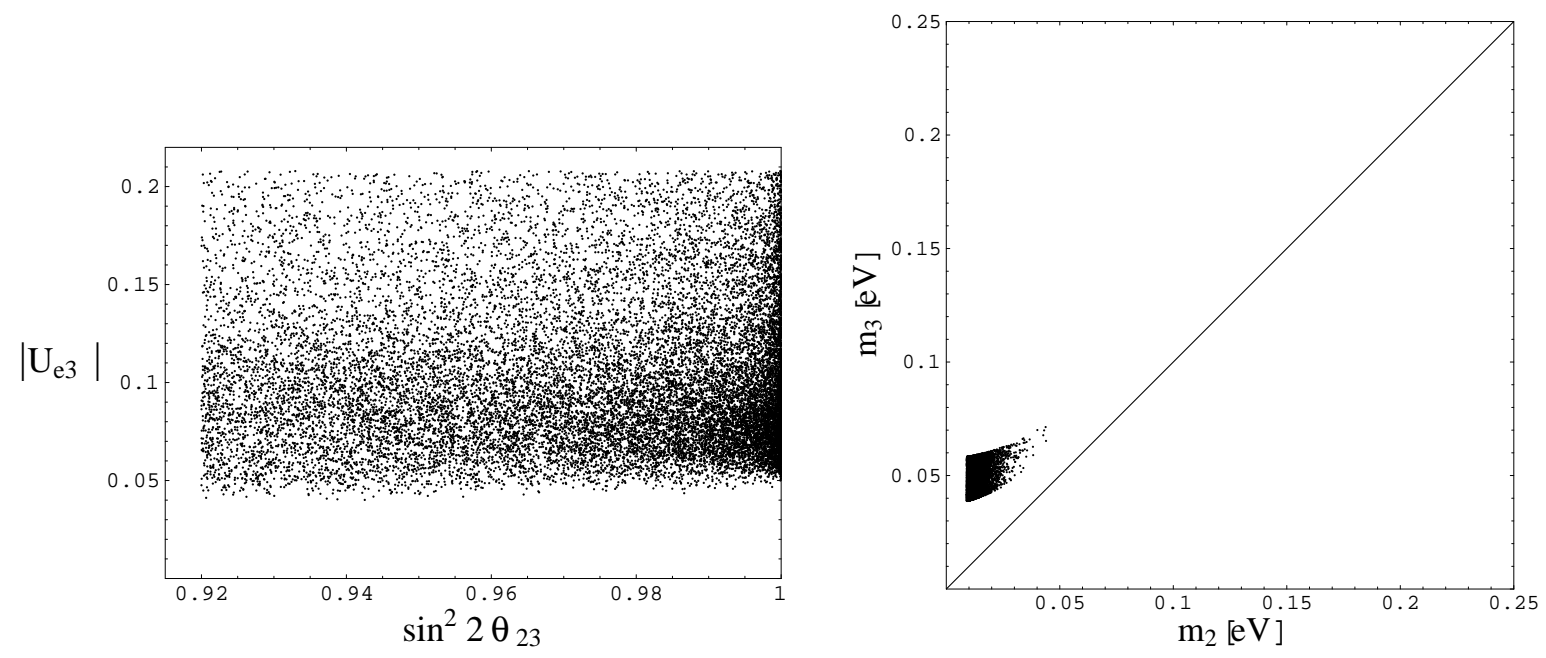

Figure 4: Scattered plot of the allowed region in the texture G-II. 

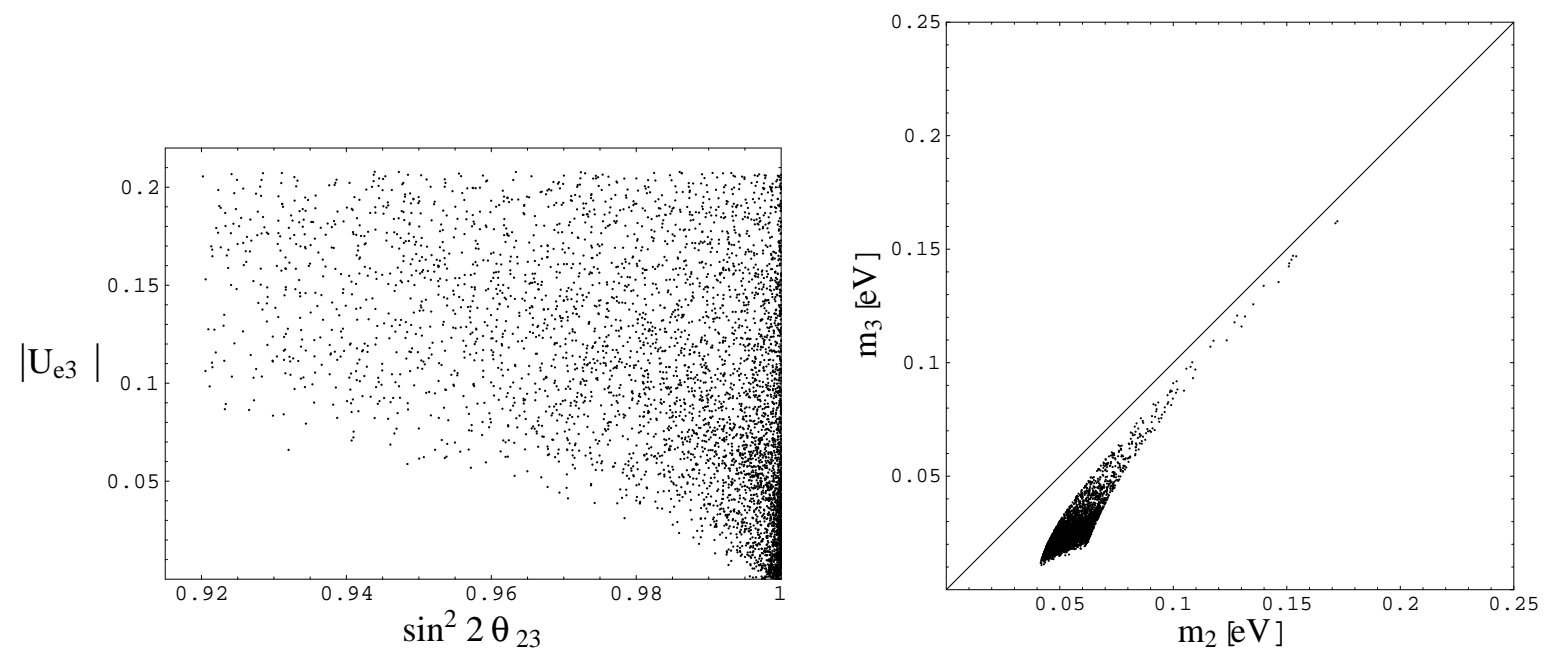

Figure 5: Scattered plot of the allowed region in the texture I-V.

Let us discuss the neutrinoless double beta decay rate, which is controlled by the effective Majorana mass:

$$
\langle m\rangle_{e e}=\left|m_{1} c_{12}^{2} c_{13}^{2} e^{i \rho}+m_{2} s_{12}^{2} c_{13}^{2} e^{i \sigma}+m_{3} s_{13}^{2} e^{-2 i \delta}\right|
$$

where $\rho=\arg \left(\lambda_{1} / \lambda_{3}\right)$ and $\sigma=\arg \left(\lambda_{2} / \lambda_{3}\right)$. This effective mass is just the absolute value of $\left(M_{\nu}\right)_{e e}$ component of the neutrino mass matrix.

The predicted lower bounds of $\langle m\rangle_{e e}$ are classified for textures in Table 4 . It is remarked that the neutrinoless double beta decay is forbidden in the textures of type IV, because of $\left(M_{\nu}\right)_{e e}=0$. The predictions of the typical textures D-V, E-II, L-II are presented in figures 6, 7 and 8.

As shown in Table 4, many hybrid textures (thirty-eight ones) predict the lower bound $10 \sim 30 \mathrm{meV}$ although there are differences of factor in the lower bound predictions for each texture. 


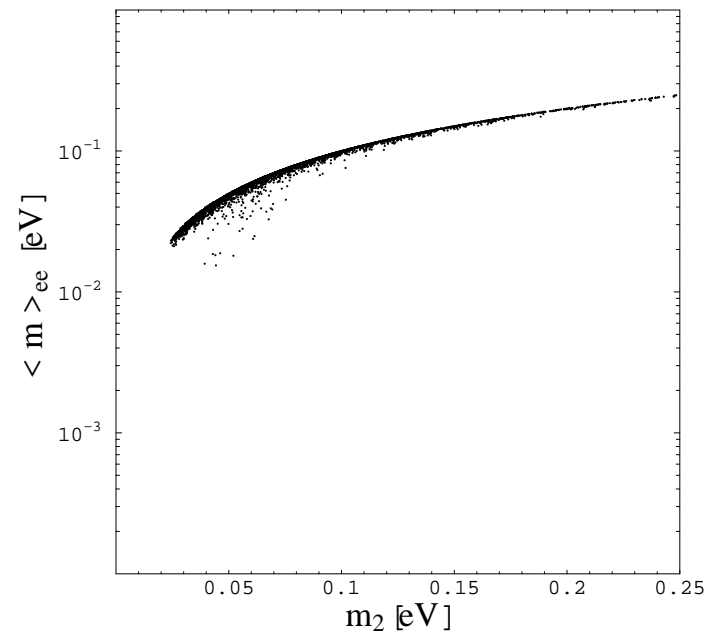

Figure 6: Neutrinoless $\beta \beta$ decays in the texture D-V.

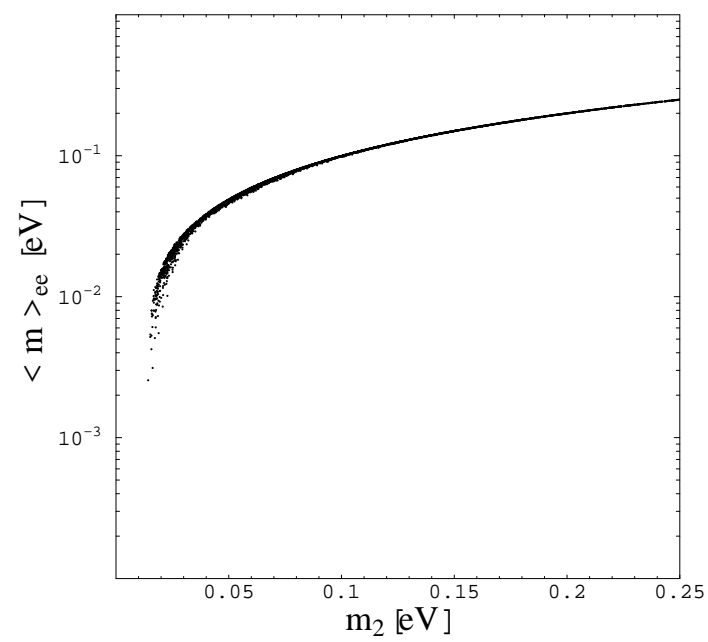

Figure 7: Neutrinoless $\beta \beta$ decays in the texture E-II. 


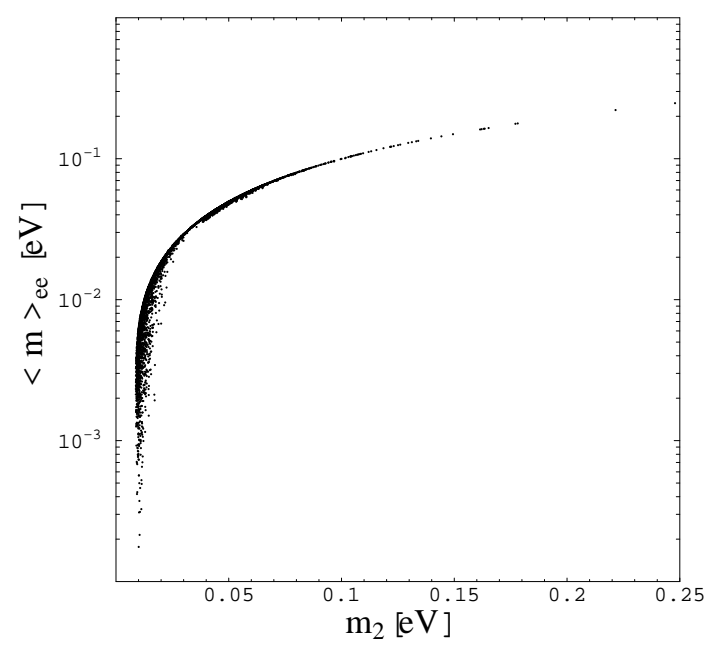

Figure 8: Neutrinoless $\beta \beta$ decays in the texture L-II.

It is remarked that there is the approximate $\mu-\tau$ flavor interchange symmetry [16] in the textures. Let us consider the following transformation:

$$
S^{T} M_{\nu} S=\hat{M}_{\nu}, \quad S=\left(\begin{array}{ccc}
1 & 0 & 0 \\
0 & 0 & 1 \\
0 & 1 & 0
\end{array}\right),
$$

which corresponds to $\theta_{23} \Leftrightarrow \frac{\pi}{2}-\theta_{23}$ interchange. Our results indicate $M_{\nu}$ and $\hat{M}_{\nu}$ are "dual". The two textures related by $S^{T} M_{\nu} S$ present almost same results in the $\sin ^{2} 2 \theta_{23}$ plot. This symmetry is due to $\theta_{23} \sim \pi / 4$ experimentally. The twenty-eight sets of the $\mu-\tau$ flavor interchange are given as

$$
\begin{array}{ll}
\mathrm{A}-\mathrm{I} \Leftrightarrow \mathrm{B}-\mathrm{II}, & \mathrm{A}-\mathrm{II} \Leftrightarrow \mathrm{B}-\mathrm{I}, \quad \mathrm{A}-\mathrm{VI} \Leftrightarrow \mathrm{B}-\mathrm{V}, \quad \mathrm{A}-\mathrm{III} \Leftrightarrow \mathrm{B}-\mathrm{III}, \\
\mathrm{L}-\mathrm{I} \Leftrightarrow \mathrm{O}-\mathrm{II}, & \mathrm{L}-\mathrm{II} \Leftrightarrow \mathrm{O}-\mathrm{I}, \quad \mathrm{L}-\mathrm{VI} \Leftrightarrow \mathrm{O}-\mathrm{V}, \quad \mathrm{L}-\mathrm{IV} \Leftrightarrow \mathrm{O}-\mathrm{IV}, \\
\mathrm{E}-\mathrm{II} \Leftrightarrow \mathrm{F}-\mathrm{I}, & \mathrm{E}-\mathrm{V} \Leftrightarrow \mathrm{F}-\mathrm{VI}, \quad \mathrm{E}-\mathrm{VI} \Leftrightarrow \mathrm{F}-\mathrm{V}, \quad \mathrm{E}-\mathrm{IV} \Leftrightarrow \mathrm{F}-\mathrm{IV}, \\
\mathrm{G}-\mathrm{II} \Leftrightarrow \mathrm{H}-\mathrm{I}, & \mathrm{G}-\mathrm{V} \Leftrightarrow \mathrm{H}-\mathrm{VI}, \quad \mathrm{G}-\mathrm{VI} \Leftrightarrow \mathrm{H}-\mathrm{V}, \quad \mathrm{G}-\mathrm{III} \Leftrightarrow \mathrm{H}-\mathrm{III}, \\
\mathrm{J}-\mathrm{II} \Leftrightarrow \mathrm{N}-\mathrm{I}, \quad \mathrm{J}-\mathrm{VI} \Leftrightarrow \mathrm{N}-\mathrm{V}, \quad \mathrm{J}-\mathrm{III} \Leftrightarrow \mathrm{N}-\mathrm{III}, \quad \mathrm{J}-\mathrm{IV} \Leftrightarrow \mathrm{N}-\mathrm{IV}, \\
\mathrm{K}-\mathrm{I} \Leftrightarrow \mathrm{M}-\mathrm{II}, \quad \mathrm{K}-\mathrm{VI} \Leftrightarrow \mathrm{M}-\mathrm{V}, \quad \mathrm{K}-\mathrm{III} \Leftrightarrow \mathrm{M}-\mathrm{III}, \quad \mathrm{K}-\mathrm{IV} \Leftrightarrow \mathrm{M}-\mathrm{IV}, \\
\mathrm{C}-\mathrm{I} \Leftrightarrow \mathrm{C}-\mathrm{II}, \quad \mathrm{D}-\mathrm{V} \Leftrightarrow \mathrm{D}-\mathrm{VI}, \quad \mathrm{I}-\mathrm{I} \Leftrightarrow \mathrm{I}-\mathrm{II}, \quad \mathrm{I}-\mathrm{V} \Leftrightarrow \mathrm{I}-\mathrm{VI},
\end{array}
$$



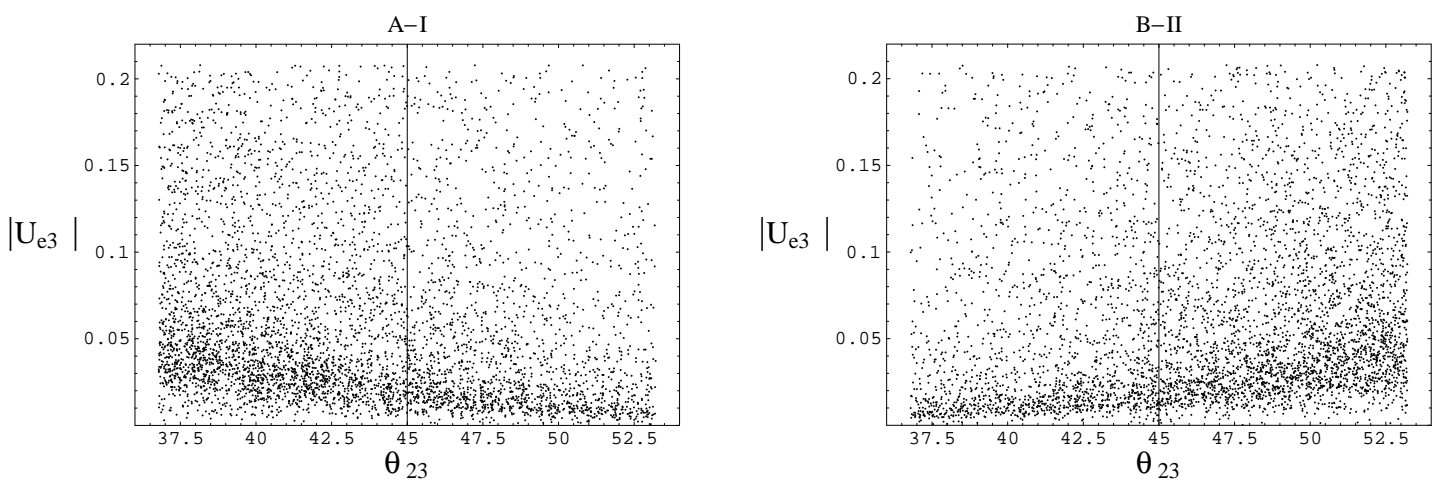

Figure 9: The interchange symmetry between A-I and B-II, which corresponds $\theta_{23} \leftrightarrow \frac{\pi}{2}-\theta_{23}$. The horizontal axis is $\theta_{23}$ [degrees].

except for

$$
\text { C-III, C-IV, D-III, D-IV, }
$$

which are transformed to themselves. In the fig. 9, we show the scattered plot on the $\theta_{23}-\left|U_{e 3}\right|$ plane for the textures of A-I and B-II, in which one can see the interchange symmetry of $\theta_{23} \leftrightarrow \frac{\pi}{2}-\theta_{23}$

\section{Summary}

We presented numerical and comprehensive analyses of the hybrid textures of neutrinos, which may be derived from the discrete symmetry. Only six textures among sixty ones are excluded by the present experimental data. Since there are many textures which give similar predictions, we classified the texture based on the numerical results. The neutrinoless double beta decay is also studied in these textures. The results may disappoint us because we cannot expect the experimental data to determine the correct texture. However, it should be emphasized that there still remain rich structure of the neutrino mass matrix in the phenomenological point of view. We expect that the cooperation between theoretical study such as the flavor symmetry and the future neutrino experiments will reveal the structure of the neutrino mass matrix. 
We are most grateful to E. Ma and M. Frigerio for appropriate advice and encouragement.

We also thanks K. Yoshioka for useful discussions. M. T is supported by the Grant-in-Aid for Science Research, Ministry of Education, Science and Culture, Japan(No.16028205). S. K is also supported by JSPS.

\section{References}

[1] Super-Kamiokande Collaboration (S. Fukuda et al.), Phys. Rev. Lett. 86 (2001) 5651, 5656 ;

SNO Collaboration (Q. R. Ahmad et al.), Phys. Rev. Lett. 87 (2001) 071301; ibid. 89 (2002) 011301; ibid. 89 (2002) 011302; ibid. 92 (2004) 181301.

[2] KamLAND Collaboration (K. Eguchi et al.), Phys. Rev. Lett. 90 (2003) 0212021;

KamLAND Collaboration (T. Araki et al.), Phys. Rev. Lett. 94 (2005) 081801.

[3] Super-Kamiokande Collaboration (Y. Fukuda et al.), Phys. Rev. Lett. 81 (1998) 1562; ibid. 82 (1999) 2644; ibid. 82 (1999) 5194.

[4] Z. Maki, M. Nakagawa and S. Sakata, Prog. Theor. Phys. 28 (1962) 870.

[5] CHOOZ Collaboration (M. Apollonio et al.), Phys. Lett. B466 (1999) 415.

[6] H. Nishiura, K. Matsuda and T. Fukuyama, Phys. Rev. D60 (1999) 013006;

E. K. Akhmedov, G. C. Branco, M. N. Rebelo, Phys. Rev. Lett. 84 (2000) 3535;

S. K. Kang and C. S. Kim, Phys. Rev. D63 (2001) 113010.

[7] P. H. Frampton, S. L. Glashow and D. Marfatia, Phys. Lett. B536 (2002) 79.

[8] Z. Xing, Phys. Lett. B530 (2002) 159.

[9] W. Guo and Z. Xing, Phys. Rev. D67 (2003) 053002;

R. Barbieri, T. Hambye and A. Romanino, JHEP 0303 (2003) 017;

A. Ibara and G. G. Ross, Phys. Lett. B575 (2003) 279;

K. T. Mahanthappa and Mu-C. Chen, Phys. Rev. D65 (2002) 053010; D68 (2003) 017301; 
M. Bando and M. Obara, Prog. Theor. Phys. 109 (2003) 995;

A. Kageyama, S. Kaneko, N. Shimoyama and M. Tanimoto, Phys. Lett. B538 (2002) 96;

S. Kaneko and M. Tanimoto, Phys. Lett. B551 (2003) 127;

M. Bando, S. Kaneko, M. Obara and M. Tanimoto, Phys. Lett. B580 (2004) 229;

M. Honda, S. Kaneko and M. Tanimoto, JHEP 0309 (2003) 028, Phys. Lett. B593 (2004) 165.

[10] M. Frigerio, S. Kaneko, E. Ma and M. Tanimoto, Phys. Rev. D71 (2005) 011901.

[11] S-L. Chen, M. Frigerio and E. Ma, Phys. Rev. D70 (2004) 073008.

[12] Y. Koide, K. Matsuda and H. Nishiura, T. Kikuchi and T. Fukuyama, Phys. Rev. D66 (2002) 093006;

Y. Koide, Phys. Rev. D69 (2004) 093001;

K. Matsuda and H. Nishiura, Phys. Rev. D69 (2004) 053005; ibid. D69 (2004) 117302;

I. Aizawa, T. Kitabayashi and M. Yasue, Phys. Rev. D71 (2005) 0705011.

[13] M. Frigerio and A. Yu. Smirnov, Phys. Rev. D67, 013007 (2003).

[14] C. Hagedorn and W. Rodejohann, hep-ph/0503143.

[15] G. L. Fogli, E. Lisi, M. Marrone, D. Montanino, A. Palazzo and A. M. Rotunno, Phys. Rev. D67 (2003) 073002;

J. N. Bahcall, M. C. Gonzalez-Garcia and C. Peña-Garay, JHEP 0302 (2003) 009;

M. Maltoni, T. Schwetz and J. W. F. Valle, Phys. Rev. D67 (2003) 093003;

P. C. Holanda and A. Yu. Smirnov, JHEP 0302 (2003) 001;

V. Barger and D. Marfatia, Phys. Lett. B555 (2003) 144;

M. Maltoni, T. Schwetz, M. Tórtola and J. W. F. Valle, Phys. Rev. D68 (2003) 113010.

[16] T. Kitabayashi and M. Yasue, Phys. Rev. D67 (2003) 015006;

C. S. Lam, Phys. Lett. B507 (2001) 214;

W. Grimus and L. Lavoura, JHEP 0107 (2001) 045; Acta Phys. Polon. B34 (2003) 5393; 
E. Ma, Phys. Rev. D66 (2002) 117301;

E. Ma and G. Rajasekaran, Phys. Rev. D68 (2003) 071302;

R. N. Mohapatra, JHEP 0410(2004) 027;

R. N. Mohapatra and S. Nasri, Phys. Rev. D71 (2005) 033001;

W. Grimus, A. S. Joshipura, S. Kaneko, L. Lavoura, H. Sawanaka and M. Tanimoto, Nucl. Phys. B713 (2005) 151. 


\begin{tabular}{|c|c|c|c|c|c|c|}
\hline & $\mathrm{I}$ & II & III & IV & $\mathrm{V}$ & $\overline{\mathrm{VI}}$ \\
\hline $\mathrm{A}$ & $\left(\begin{array}{lll}X & 0 & e \\
& X & f \\
& & c\end{array}\right)$ & $\left(\begin{array}{lll}X & d & 0 \\
& X & f \\
& & c\end{array}\right)$ & $\left(\begin{array}{lll}X & d & e \\
& X & 0 \\
& & c\end{array}\right)$ & two zeros & two zeros & $\left(\begin{array}{lll}X & d & e \\
& X & f \\
& & 0\end{array}\right)$ \\
\hline B & $\left(\begin{array}{ccc}X & 0 & e \\
& b & f \\
& & X\end{array}\right)$ & $\left(\begin{array}{lll}X & d & 0 \\
& b & f \\
& & X\end{array}\right)$ & $\left(\begin{array}{lll}X & d & e \\
& b & 0 \\
& & X\end{array}\right)$ & two zeros & $\left(\begin{array}{ccc}X & d & e \\
& 0 & f \\
& & X\end{array}\right)$ & two zeros \\
\hline $\mathrm{C}$ & $\left(\begin{array}{ccc}a & 0 & e \\
& X & f \\
& & X\end{array}\right)$ & $\left(\begin{array}{lll}a & d & 0 \\
& X & f \\
& & X\end{array}\right)$ & $\left(\begin{array}{ccc}a & d & e \\
& X & 0 \\
& & X\end{array}\right)$ & $\left(\begin{array}{lll}0 & d & e \\
& X & f \\
& & X\end{array}\right)$ & two zeros & two zeros \\
\hline $\mathrm{D}$ & two zeros & two zeros & $\left(\begin{array}{ccc}a & X & X \\
& b & 0 \\
& & c\end{array}\right)$ & $\left(\begin{array}{ccc}0 & X & X \\
& b & f \\
& & c\end{array}\right)$ & $\left(\begin{array}{ccc}a & X & X \\
& 0 & f \\
& & c\end{array}\right)$ & $\left(\begin{array}{ccc}a & X & X \\
& b & f \\
& & 0\end{array}\right)$ \\
\hline $\mathrm{E}$ & two zeros & $\left(\begin{array}{ccc}a & X & 0 \\
& b & X \\
& & c\end{array}\right)$ & two zeros & $\left(\begin{array}{ccc}0 & X & e \\
& b & X \\
& & c\end{array}\right)$ & $\left(\begin{array}{ccc}a & X & e \\
& 0 & X \\
& & c\end{array}\right)$ & $\left(\begin{array}{ccc}a & X & e \\
& b & X \\
& & 0\end{array}\right)$ \\
\hline $\mathrm{F}$ & $\left(\begin{array}{ccc}a & 0 & X \\
& b & X \\
& & c\end{array}\right)$ & two zeros & two zeros & $\left(\begin{array}{ccc}0 & d & X \\
& b & X \\
& & c\end{array}\right)$ & $\left(\begin{array}{ccc}a & d & X \\
& 0 & X \\
& & c\end{array}\right)$ & $\left(\begin{array}{ccc}a & d & X \\
& b & X \\
& & 0\end{array}\right)$ \\
\hline G & two zeros & $\left(\begin{array}{ccc}X & X & 0 \\
& b & f \\
& & c\end{array}\right)$ & $\left(\begin{array}{ccc}X & X & e \\
& b & 0 \\
& & c\end{array}\right)$ & two zeros & $\left(\begin{array}{ccc}X & X & e \\
& 0 & f \\
& & c\end{array}\right)$ & $\left(\begin{array}{ccc}X & X & e \\
& b & f \\
& & 0\end{array}\right)$ \\
\hline $\mathrm{H}$ & $\left(\begin{array}{ccc}X & 0 & X \\
& b & f \\
& & c\end{array}\right)$ & two zeros & $\left(\begin{array}{ccc}X & d & X \\
& b & 0 \\
& & c\end{array}\right)$ & two zeros & $\left(\begin{array}{ccc}X & d & X \\
& 0 & f \\
& & c\end{array}\right)$ & $\left(\begin{array}{ccc}X & d & X \\
& b & f \\
& & 0\end{array}\right)$ \\
\hline I & $\left(\begin{array}{ccc}X & 0 & e \\
& b & X \\
& & c\end{array}\right)$ & $\left(\begin{array}{ccc}X & d & 0 \\
& b & X \\
& & c\end{array}\right)$ & two zeros & two zeros & $\left(\begin{array}{ccc}X & d & e \\
& 0 & X \\
& & c\end{array}\right)$ & $\left(\begin{array}{ccc}X & d & e \\
& b & X \\
& & 0\end{array}\right)$ \\
\hline $\mathrm{J}$ & two zeros & $\left(\begin{array}{ccc}a & X & 0 \\
& X & f \\
& & c\end{array}\right)$ & $\left(\begin{array}{ccc}a & X & e \\
& X & 0 \\
& & c\end{array}\right)$ & $\left(\begin{array}{lll}0 & X & e \\
& X & f \\
& & c\end{array}\right)$ & two zeros & $\left(\begin{array}{ccc}a & X & e \\
& X & f \\
& & 0\end{array}\right)$ \\
\hline $\mathrm{K}$ & $\left(\begin{array}{ccc}a & 0 & X \\
& X & f \\
& & c\end{array}\right)$ & two zeros & $\left(\begin{array}{ccc}a & d & X \\
& X & 0 \\
& & c\end{array}\right)$ & $\left(\begin{array}{ccc}0 & d & X \\
& X & f \\
& & c\end{array}\right)$ & two zeros & $\left(\begin{array}{ccc}a & d & X \\
& X & f \\
& & 0\end{array}\right)$ \\
\hline $\mathrm{L}$ & $\left(\begin{array}{ccc}a & 0 & e \\
& X & X \\
& & c\end{array}\right)$ & $\left(\begin{array}{ccc}a & d & 0 \\
& X & X \\
& & c\end{array}\right)$ & two zeros & $\left(\begin{array}{ccc}0 & d & e \\
& X & X \\
& & c\end{array}\right)$ & two zeros & $\left(\begin{array}{ccc}a & d & e \\
& X & X \\
& & 0\end{array}\right)$ \\
\hline M & two zeros & $\left(\begin{array}{ccc}a & X & 0 \\
& b & f \\
& & X\end{array}\right)$ & $\left(\begin{array}{ccc}a & X & e \\
& b & 0 \\
& & X\end{array}\right)$ & $\left(\begin{array}{ccc}0 & X & e \\
& b & f \\
& & X\end{array}\right)$ & $\left(\begin{array}{ccc}a & X & e \\
& 0 & f \\
& & X\end{array}\right)$ & two zeros \\
\hline $\mathrm{N}$ & $\left(\begin{array}{ccc}a & 0 & X \\
& b & f \\
& & X\end{array}\right)$ & two zeros & $\left(\begin{array}{ccc}a & d & X \\
& b & 0 \\
& & X\end{array}\right)$ & $\left(\begin{array}{ccc}0 & d & X \\
& b & f \\
& & X\end{array}\right)$ & $\left(\begin{array}{ccc}a & d & X \\
& 0 & f \\
& & X\end{array}\right)$ & two zeros \\
\hline $\mathrm{O}$ & $\left(\begin{array}{ccc}a & 0 & e \\
& b & X \\
& & X\end{array}\right)$ & $\left(\begin{array}{ccc}a & d & 0 \\
& b & X \\
& & X\end{array}\right)$ & two zeros & $\left(\begin{array}{lll}0 & d & e \\
& b & X \\
& & X\end{array}\right)$ & $\left(\begin{array}{ccc}a & d & e \\
& 0 & X \\
& & X\end{array}\right)$ & two zeros \\
\hline
\end{tabular}

Table 1: Sixty hybrid textures. $X$ denotes two equal elements in all textures. 


\begin{tabular}{|c|c|c|c|c|c|c|}
\hline & I & II & III & IV & V & VI \\
\hline A & NH + DL & NH + DL & IH + DL & - & - & ALL \\
\hline B & NH + DL & NH + DL & IH + DL & - & ALL & - \\
\hline C & ALL & ALL & IH + DL & NH & - & - \\
\hline D & - & - & IH + DL & NH & ALL & ALL \\
\hline E & - & ALL & - & $\times$ & IH + DL & IH \\
\hline F & ALL & - & - & $\times$ & IH & IH + DL \\
\hline G & - & NH & IH & - & ALL & IH + DL \\
\hline H & NH & - & IH & - & IH + DL & ALL \\
\hline I & NH + DL & NH + DL & - & - & IH + DL & IH + DL \\
\hline J & - & ALL & IH & $\times$ & - & ALL \\
\hline K & ALL & - & IH & $\times$ & - & ALL \\
\hline L & ALL & ALL & - & NH & - & IH \\
\hline M & - & ALL & IH & $\times$ & ALL & - \\
\hline N & ALL & - & IH & $\times$ & ALL & - \\
\hline O & ALL & ALL & - & NH & IH & - \\
\hline
\end{tabular}

Table 2: Mass patterns for each texture. NH, IH and DL denote the normal hierarchy, the inverted one, and the degenerate one, respectively. ALL denotes $\mathrm{NH}+\mathrm{IH}+\mathrm{DL}$, and $\times$ means that the texture is excluded by the present experimental data.

\begin{tabular}{|c|c|c|c|c|c|}
\hline A-I & C-III & C-IV & G-II & I-V \\
\hline$\left(\begin{array}{lll}1 & 0 & \lambda \\
0 & 1 & 1 \\
\lambda & 1 & 1\end{array}\right)$ & $\left(\begin{array}{lll}1 & 1 & 1 \\
1 & 1 & 0 \\
1 & 0 & 1\end{array}\right)$ & $\left(\begin{array}{ccc}0 & \lambda & \lambda \\
\lambda & 1 & 1 \\
\lambda & 1 & 1\end{array}\right)$ & $\left(\begin{array}{ccc}\lambda & \lambda & 0 \\
\lambda & 1 & 1 \\
0 & 1 & 1\end{array}\right)$ & $\left(\begin{array}{ccc}1 & 1 & 1 \\
1 & 0 & 1 \\
1 & 1 & \lambda\end{array}\right)$ \\
\hline
\end{tabular}

Table 3: The relative magnitudes of the matrix elements in the unit of $\lambda \simeq 0.2$. 


\begin{tabular}{|c|r|c|c|}
\hline $10 \sim 30 \mathrm{meV}$ & $1 \sim 3 \mathrm{meV}$ & $0.1 \sim 0.3 \mathrm{meV}$ & 0 \\
\hline Others & C-I, C-II, E-II, F-I, G-II, H-I, J-II, N-I & L-I, L-II, O-I, O-II & all-IV \\
\hline
\end{tabular}

Table 4: The classification of the predicted lower bounds of $\langle m\rangle_{e e}$. 